\title{
Clinical Outcomes and Lens Constant Optimization of the Zeiss CT Lucia 602 Lens Using a Modified Yamane Technique
}

This article was published in the following Dove Press journal: Clinical Ophthalmology

\author{
Edward L Randerson' \\ Joseph D Bogaard' \\ Lisa R Koenig $\mathbb{D}^{2}$ \\ Eileen S Hwang $\mathbb{D}^{3}$ \\ Clinton C Warren' \\ Steven B Koenig (D) \\ 'Department of Ophthalmology, \\ Froedtert and the Medical College of \\ Wisconsin, Milwaukee, WI, USA; ${ }^{2}$ Weill \\ Cornell Medical College, New York, NY, \\ USA; ${ }^{3}$ Department of Ophthalmology \& \\ Visual Sciences, University of Utah, Salt \\ Lake City, UT, USA
}

Purpose: To report the clinical and refractive outcomes of a modified Yamane technique for scleral fixation of the CT Lucia 602 lens.

Design: Retrospective case series.

Patients: One hundred twenty-one eyes with dislocated posterior chamber lens implants, surgical aphakia, subluxed crystalline lenses, capsular tear, anterior chamber, or iris sutured posterior chamber lens intolerance were included.

Methods: Secondary implantation of the Zeiss CT Lucia 602 lens was performed by a single surgeon using a modified Yamane technique employing a single needle, rather than the double-needle approach. One hundred twelve eyes underwent simultaneous 3 port pars plana vitrectomy and 9 eyes had previously undergone posterior vitrectomy surgery. Exclusion criteria were age $<18$, simultaneous glaucoma or corneal procedures, staged corneal transplantation, and follow-up $<30$ days.

Main Outcome Measures: A paired $t$-test was used to compare pre- and post-operative corrected distance visual acuity (CDVA), the mean predictive error, and the mean absolute predictive error between the manufacturer's and optimized constants for Hoffer Q, Holladay I, and SRK/T. Complication rates were reported.

Results: One hundred twenty-one consecutive eyes with a mean follow-up of 237 days were included. The pre-operative CDVA was $0.871 \pm 0.785 \operatorname{logMAR}$ (mean \pm standard deviation, Snellen equivalent 20/149), which improved to $0.401 \pm 0.608$ (Snellen equivalent 20/50) postoperatively. In 109 eyes with reliable postoperative refractions, the mean predictive refractive error (D) \pm std was $+0.74 \pm 1.37$ for Hoffer Q, $+0.66 \pm 1.41$ for Holladay 1 , and $+0.47 \pm 1.49$ for SRK/T $(p<0.05)$. Refractive outcome analysis yielded a mean optimized personalized anterior chamber depth (pACD) of 5.69, Surgeon Factor of 1.79, and A constant of 118.56. Vision-limiting complications occurred in 11 eyes $(9.1 \%)$.

Conclusion: Scleral fixation of the CT Lucia 602 lens using a single-needle modification of the Yamane technique resulted in very good visual acuity, predictable postoperative refractive errors, but some vision-limiting complications in this heterogeneous group of eyes with significant comorbidities.

Keywords: Yamane technique, scleral lens fixation, secondary lens implantation, sutureless IOL fixation

\section{Introduction}

Secondary intraocular lens (IOL) implantation in the absence of capsular support provides a unique surgical challenge. Earlier used methods of IOL support include fixation using an anterior chamber intraocular lens (ACIOL), an iris-fixated
Correspondence: Steven B Koenig

Tel + I 414 228-1653

Email sbkoenig52@gmail.com 
posterior chamber intraocular lens (iris-fixated PCIOL), or a trans-scleral sutured PCIOL. ${ }^{1}$ While successful, each technique has notable disadvantages and complicationsACIOLs have been associated with increased endothelial cell loss, peripheral anterior synechiae, as well as uveitisglaucoma-hyphema (UGH) syndrome; iris-fixated PCIOLs have been associated with chaffing of the posterior pigmented epithelium, subsequent pigment dispersion, UGH syndrome and late suture breakage, and may interfere with pupil dilation; trans-scleral sutured PCIOLs have been associated with postoperative hypotony, suture erosion with endophthalmitis, and late suture breakage leading to lens dislocation. ${ }^{2-10}$ Sutureless techniques for intrascleral PCIOL fixation have also been described using scleral tunnels. ${ }^{11-14}$ Recently, Yamane et al described a sutureless technique for intrascleral fixation of a posterior chamber lens using ab externo sclerotomies, simultaneous externalization of the lens haptics using two separate needles, and thermocauterization to flange the distal haptics with intrascleral fixation. ${ }^{15}$ Using this technique, they have reported excellent visual results, predictable postoperative refractive errors, low endothelial cell loss, and relatively few complications. ${ }^{15}$ The Yamane technique has gained popularity, and several modifications have been described. ${ }^{16}$ We modified Yamane's technique by fixating the leading haptic first, ${ }^{17}$ which avoids the need for an assistant or special device to hold the first needle during docking of the second needle. ${ }^{18}$

Refractive outcomes are expected to differ in scleral fixation of IOLs compared to endocapsular IOL placement because the precise coordinates of the scleral opening is a major determinant of estimated lens position. ${ }^{19}$ Information on refractive outcomes of the CT Lucia 602 lens is necessary in order to improve the predictability of the Yamane technique, yet information is limited. We did a literature review using PubMed from January 1, 2017 to May 1, 2020 and found 2 publications that reported the difference between refractive error predicted by IOL formulas and actual post-op refraction. However, both studies combined information from multiple different IOLs, precluding lens constant optimization. ${ }^{15,20}$

We report the clinical outcomes of a series of 121 consecutive eyes of a heterogeneous population that underwent implantation of the Zeiss CT Lucia 602 lens (formerly the Aaren Scientific EC3PAL) by a single surgeon using the single-needle modification of the Yamane technique. In 109 eyes with reliable postoperative refractions, we were able to optimize the lens constant, which is necessary to improve refractive outcomes using the Yamane technique.

\section{Methods}

A retrospective chart review was conducted in accordance with the tenets of the Declaration of Helsinki. Approval of the Institutional Review Board of the Medical College of Wisconsin was obtained. Informed consent was obtained from all patients by the operating surgeons before the surgical procedure. Surgical indications for a secondary scleral fixated intraocular lens (SFIOL) included: surgical aphakia without adequate capsular support, ACIOL intolerance with subsequent UGH syndrome and/or intractable cystoid macular edema, crystalline lens subluxation, PCIOL dislocation or malposition. Exclusion criteria were: (1) patients under the age of eighteen (2) patients undergoing planned staged intraocular lens and corneal transplant procedures (3) patients undergoing combined SFIOL placement with glaucoma or corneal procedures (4) patients with postoperative follow up of fewer than 30 days.

All patients underwent standard pre- and postoperative ophthalmic examinations including: manifest refraction with corrected distance visual acuity (CDVA), slit-lamp examination, dilated fundus examination, measurement of intraocular pressure using either a Tono-Pen (Reichert Technologies, Buffalo, New York) or Goldmann applanation tonometer, automated keratometry and axial length measurements using either the IOL Master (Zeiss, Carl Zeiss Meditec, Jena, Germany) or the AccuSonic A-Scan (Accutome, Malvern, Pennsylvania) adjusting for aphakia if the existing lens implant was dislocated outside the visual axis. The manufacturer's recommended A constant of 117.7 was used for the CT Lucia 602 lens implant in all patients. The Holliday I formula was used to calculate the IOL power in eyes with an axial length of $22-25 \mathrm{~mm}$, the SRK/T formula was used for longer eyes $(>25 \mathrm{~mm})$, and the Hoffer Q formula was used for shorter eyes $(<22 \mathrm{~mm})$. All SFIOLs included were implanted by a single surgeon (SBK) at the Eye Institute of Froedtert Hospital and the Medical College of Wisconsin between 7/ $27 / 17$ and 3/2/2020. Chart review was completed on all patients by $4 / 18 / 2020$.

\section{IOL Calculations and Statistical Methods}

Twelve eyes with unobtainable or unreliable subjective postoperative refractions were excluded from refractive outcome analysis, but were included in the visual acuity results. Using the original formulas and subsequent updates/errata for Holladay I, Hoffer Q, and SRK/T, an A constant, Surgeon 
Factor (SF), and personalized anterior chamber depth (pACD) were directly calculated for each eye from preoperative axial length and K's, the implanted IOL power, and the final spherical equivalent refraction using Excel 2016 (Microsoft). ${ }^{21-23}$ The three constants obtained from each individual eye were then averaged to obtain the optimized constants. The raw data and calculations were reanalyzed and confirmed by another independent source.

The prediction error for each eye was then calculated for each of the formulas using the manufacturer's constants and the optimized constants as the post-operative refractive error minus the expected error (in spherical equivalent diopters). A paired $t$-test was used to compare pre- and post-operative CDVA, the mean predictive error, and the mean absolute predictive error between the manufacturer's and optimized constants for capsular bag placement with Hoffer Q, Holladay I, SRK/T using Excel 2016 (Microsoft).

\section{Surgical Procedure}

All surgeries were performed under either retrobulbar with O'Brien lid block, or sub-Tenon's anesthesia using $2 \%$ lidocaine hydrochloride. With the exception of 9 patients who had previously undergone a thorough pars plana vitrectomy, surgeries were performed together with vitreoretinal faculty. First, a 3-port pars plana vitrectomy was completed. Retrieval and removal of a dislocated PCIOL implant and capsular bag/Sommering ring, pars plana lensectomy of a subluxed crystalline lens, or removal of an ACIOL were completed when necessary prior to beginning SFIOL insertion. If the PCIOL to be removed was composed of silicone acrylate or silicone, the lens optic was nearly completely transected with an IOL cutter and rotated from the eye using an MST forceps (MicroSurgical Technology, Redmond, Washington) through a $3 \mathrm{~mm}$ clear corneal incision-commonly referred to as "Pacman technique". ACIOLs and PCIOLs composed of polymethylmethacrylate were removed intact through a $6 \mathrm{~mm}$ clear corneal incision which was subsequently closed with interrupted buried $10-0$ nylon stitches. Whenever possible, the original cataract incision was used.

The surgical procedure has been previously described (Video 1). ${ }^{17}$ Postoperatively, the eyes were treated with topical ofloxacin $0.3 \%$ (Ocuflox) or ciprofloxacin $0.3 \%$ (Ciloxan) four times daily for 1 week and prednisolone acetate $1 \%$ four times daily for 1 month. When used for large incisions, corneal sutures were removed around postoperative month 3 with subsequent measurement of final refraction and CDVA. Additionally, if patients experienced cystoid macular edema at the post-operative month 1 visit, the course of prednisolone acetate $1 \%$ was continued and their CDVA was rechecked at resolution around postoperative month $2-3$.

\section{Results}

One-hundred and twenty-one eyes with an average age of 68.2 years (range 21-95) were included with an average follow-up time of 237 days (range 30-1079). Eighty-one eyes had follow-up greater than 3 months and 30 eyes had a postoperative interval of at least 1 year. Patient characteristics are included in Table 1. From most common to least, the pre-operative indication for secondary IOL included dislocated PCIOL in 65 eyes (53.7\%), aphakia in 38 eyes $(31.4 \%)$, dislocated/subluxed crystalline lens in 12 eyes $(9.9 \%)$, intolerance of an ACIOL in 4 eyes $(3.3 \%)$, and "other" in 2 eyes (1.7\%) including 1 eye $(0.8 \%)$ with phakic posterior capsular rupture following unrelated vitrectomy and 1 eye $(0.8 \%)$ with intolerance of an iris sutured PCIOL. Notably, 28 eyes (23.1\%) had a prior ocular history of facial trauma with $6(5.0 \%)$ having prior ruptured globe and 5 (4.1\%) having prior intraocular foreign body. Other complicating past ocular history $(\mathrm{POH})$ is included in Table 2. The average pre-operative corrected $\log$ MAR distance visual acuity was $0.871 \pm 0.785$ (Snellen equivalent of 20/149), improving to $0.401 \pm 0.608$ (Snellen equivalent of 20/50) post-operatively $(p<$ $0.0001)$. This was largely due to removal of a dislocated posterior chamber lens and opacified capsular complex obscuring the visual axis, resolution of pre-existing cystoid macular edema, resolution of corneal edema or removal of a cataractous crystalline lens. Admittedly, accurate preoperative manifest refraction and visual acuity may be difficult to obtain, particularly in eyes with subluxed lens implants or crystalline lenses, eyes with keratoconus or corneal disease and eyes with preoperative macular disease. In light of the extensive pre-operative ocular comorbidities, the average post-operative visual acuity improved significantly.

The most common post-operative complication was transient elevated intraocular pressure (IOP) greater than $25 \mathrm{mmHg}$, which was found in 19 eyes (15.7\%); each case resolved with the application of IOP-lowering medications or in-office anterior chamber paracentesis. The second most common complication was exposed or recurrent protrusion of the flanged haptic, occurring in 11 eyes $(9.1 \%)$; with the exception of two eyes (discussed below), the 
Table I Patient Characteristics

\begin{tabular}{|c|c|}
\hline & Eyes \\
\hline Number of Eyes & 121 \\
\hline Mean Age (years) & $68.17(21-95)$ \\
\hline$\%$ Male & 61.16 \\
\hline \% Right Eye & 56.20 \\
\hline Mean F/U Time (days) & $236.98(30-1079)$ \\
\hline Mean Axial Length (mm) & $24.77 \pm 1.79$ \\
\hline \multicolumn{2}{|l|}{ Diagnosis } \\
\hline Aphakia & 38 \\
\hline Dislocated PCIOL & 65 \\
\hline Dislocated/Unstable Crystalline lens & 12 \\
\hline Intolerant ACIOL & 4 \\
\hline Other & 2 \\
\hline Trauma Related & 28 \\
\hline Complicated Cataract Surgery & 26 \\
\hline \multicolumn{2}{|l|}{ Visual Acuity } \\
\hline Preop CDVA (logMAR) & $0.87 \mid \pm 0.785$ \\
\hline Preop CDVA (Snellen equivalent) & $20 / 149$ \\
\hline Postop CDVA (logMAR) & $0.401 \pm 0.608$ \\
\hline Postop CDVA (Snellen equivalent) & $20 / 50$ \\
\hline \multicolumn{2}{|l|}{ Refractive Outcomes } \\
\hline Mean difference from target $(D) \pm$ STD & $0.183 \pm 1.449$ \\
\hline Median difference from target (D) & 0.125 \\
\hline Mean absolute difference from target $(D) \pm$ STD & $1.025 \pm 1.035$ \\
\hline Median absolute difference from target (D) & 0.625 \\
\hline
\end{tabular}

haptics were successfully repositioned at the slit lamp. Cystoid macular edema and corneal edema occurred in 7 eyes $(5.8 \%)$ and 6 eyes $(5.0 \%)$, respectively; in all of these cases, symptoms resolved with a prolonged course of topical steroids in conjunction with topical non-steroidal anti-inflammatory drops titrated from 1 to 4 times per day. Several complications occurred uncommonly: spontaneously clearing vitreous hemorrhage was seen in 4 eyes (3.3\%); spontaneously clearing hyphema occurred in 2 eyes $(1.65 \%)$; transient post-operative hypotony was seen in 4 eyes $(3.3 \%)$; and reverse pupillary block developed in 4 eyes $(3.3 \%)$.

Vision-limiting or severe complications occurred in 11 eyes $(9.1 \%)$, of which 6 eyes $(5.0 \%)$ required additional surgery: 2 eyes $(1.7 \%)$ with recurrent haptic protrusion and subsequent exposure despite repositioning in clinic, 1 eye $(0.8 \%)$ with corneal failure secondary to anterior migration of a $0.7 \mathrm{mg}$ dexamethasone implant (OZURDEX, Allergan) that required unplanned Descemet stripping automated endothelial keratoplasty (DSAEK), 1 eye $(0.8 \%)$ with
Table 2 Past Ocular History

\begin{tabular}{|c|c|}
\hline & Eyes \\
\hline Uveitis & 6 \\
\hline Trauma & 11 \\
\hline Ruptured Globe & 6 \\
\hline Intraocular Foreign Body & 5 \\
\hline Glaucoma & 32 \\
\hline Prior Glaucoma Surgery & 7 \\
\hline Corneal disease & 19 \\
\hline Band Keratopathy & 3 \\
\hline Pseudophakic Bullous Keratopathy & 2 \\
\hline Corneal Edema & 1 \\
\hline Fuch's Dystrophy & 1 \\
\hline Keratoconus & 4 \\
\hline PKP & 4 \\
\hline Herpetic Keratopathy & 1 \\
\hline Corneal Scar & 5 \\
\hline Other & 3 \\
\hline Refractive Surgery & 3 \\
\hline Pseudoexfoliation without glaucoma & 10 \\
\hline Retinal disease & 54 \\
\hline Endophthalmitis & 2 \\
\hline Macular/Myopic Degeneration/Central Drusen & 7 \\
\hline Central Macular Edema & 5 \\
\hline Epiretinal Membrane/Macular Hole & 18 \\
\hline Branch Retinal Vein Occlusion & 1 \\
\hline Vitreous Hemorrhage & 5 \\
\hline Retinal Tears & 1 \\
\hline Acute Retinal Necrosis & 1 \\
\hline Proliferative Diabetic Retinopathy & 2 \\
\hline Diabetic Macular Edema & I \\
\hline Retinal Detachment & 28 \\
\hline Proliferative Vitreoretinopathy & 5 \\
\hline Choroidal Hemorrhage & 1 \\
\hline Congenital/Inherited & 7 \\
\hline
\end{tabular}

retinal detachment that required additional pars plana vitrectomy and repair, 1 eye $(0.8 \%)$ with significant $\mathrm{POH}$ of ruptured globe with recurrent retinal detachment with proliferative vitreoretinopathy developed atrophia bulbi and count fingers vision postoperatively, and 1 eye $(0.8 \%)$ with severe choroidal hemorrhage resulting in no light perception vision despite choroidal drainage. One eye $(0.8 \%)$, in a 93-year-old patient with a $\mathrm{POH}$ of corneal edema secondary to complicated cataract extraction, developed persistent corneal edema following SFIOL implantation and elected not to proceed with DSAEK - final CDVA was 
$1.824(3 / 200)$. Three eyes (2.5\%) had decreased visual acuity due to intraocular lens tilt or decentration. One eye $(0.8 \%)$ developed endophthalmitis without haptic exposure on post-operative day 5 due to gram positive cocci treated by vitreous tap and injection of pharmacy-compounded vancomycin $1 \mathrm{mg}$ in $0.5 \mathrm{~mL}$ and ceftazidime $2.5 \mathrm{mg}$ in $0.5 \mathrm{~mL}$ - final CDVA was 0.0969 (20/25). All complications are listed in Table 3.

Twelve eyes (9.9\%) with unreliable postoperative refractions were excluded from the refractive outcome assessment, but were included in the visual acuity results. An unpaired Student's $t$-test was performed on each of the categories in Table 1; there were no statistically significant differences $(p<0.05)$ in the subgroup from the overall group. Table 4 demonstrates each formula's $0.5 \mathrm{D}, 1 \mathrm{D}$ and 2D predictive accuracy, mean predictive error, and mean absolute predictive error. Our mean optimized pACD was 5.69, Surgeon Factor was 1.79, and A constant was 118.56. The median optimized constants were also calculated: $\mathrm{pACD}=5.54$, Surgeon Factor $=1.55$ and $\mathrm{A}$ constant $=118.44$ (Table 5). The median optimized pACD and Surgeon Factor yielded better results for the percentage of eyes predicted to fall within $\pm 0.50 \mathrm{D}$, while the mean optimized A constant yielded slightly better predictability for the same target refraction. The median optimized pACD and Surgeon Factor were less accurate in predicting the postoperative refraction within \pm 1.00 D. Our data are summarized in Figure 1 as a scatter plot comparing the axial length of each eye with the calculated Surgeon factor (A), A constant (B), or pACD (C). We

Table 3 Post Operative Complications

\begin{tabular}{|l|l|}
\hline & Eyes \\
\hline None & 63 \\
Elevated IOP $(\mathrm{IOP}>25 \mathrm{mmHg})$ & 19 \\
Hypotony (IOP $<6 \mathrm{mmHg})$ & 5 \\
Hyphema & 2 \\
Reverse Pupillary Block & 4 \\
Exposed Haptic & $\mathrm{II}$ \\
Cystoid Macular Edema & 7 \\
Vitreous Hemorrhage & 4 \\
Persistent Corneal Edema & 2 \\
Severe Choroidal Hemorrhage & 1 \\
Retinal Detachment & 1 \\
Endophthalmitis & 1 \\
Visually Significant Lens Tilt or Decentration & 6 \\
Requiring Additional Surgery & 6 \\
\hline
\end{tabular}

compared the refractive outcomes, visual acuity outcomes, and lens constant optimizations for each formula and found no statistical difference between eyes with more or less than 6 months follow-up ( $\mathrm{p}>0.05$ for all comparisons).

\section{Discussion}

Yamane et al have described an elegant technique of securing a PCIOL using transconjunctival intrascleral fixation. ${ }^{15}$ Briefly, their technique involves two ab externo sclerotomies with two separate needles followed by sequential docking of the lens haptics and simultaneous double-needle withdrawal to externalize the haptics for thermocautery flanging and subsequent scleral fixation. Their study included 100 consecutive eyes and with 4 different IOL models (Santen X-70, Abbott Medical Optics ZA9003, Kowa PN6A, and Alcon MA60MA), and reported a significant improvement in mean postoperative visual acuity at 6 months compared to preoperative visual acuity, with a small average loss of corneal endothelial cells (97 cells $/ \mathrm{mm}^{2}$ ) over 3 years. Using swept-source optical coherence tomography, they found a mild degree of lens tilt (average 3.4 degrees) and a mean refractive difference from that predicted by the $\mathrm{SRK} / \mathrm{T}$ formula of $-0.21 \pm 0.99$ diopters. The most common postoperative complications included iris capture by the lens (8\%), vitreous hemorrhage $(5 \%)$, and late elevation of intraocular pressure (1\%) due to pigment dispersion. Transient ocular hypotony (2\%) and corneal edema (1\%) occurred in the early postoperative period. There were no cases of retinal detachment, choroidal hemorrhage, atrophia bulbi, endophthalmitis, or IOL dislocation in their series. ${ }^{15}$

Although Yamane et al have demonstrated outstanding results, their technique remains surgically challenging for a single surgeon. By using two separate needles for the $a b$ externo sclerotomies, the surgeon either needs an assistant to stabilize the first needle, or must allow the first syringe to lie on the surface of the globe with the tip of the needle at risk of damaging the peripheral retina, pars plicata, or posterior iris. In addition, if the lens haptics disinsert from either needle lumen during simultaneous double-needle withdrawal, the lens could dislocate posteriorly into the vitreous cavity. We feel our modification of Yamane's technique simplifies the procedure for a single surgeon: it provides optic-haptic stability during a sequential, rather than simultaneous, single-needle withdrawal. This sequence of trailing haptic docking in the clear corneal wound followed by individual haptic externalization and 
Table 4 IOL Calculation and Optimization

\begin{tabular}{|c|c|c|c|}
\hline Results with Standard Constants & Hoffer $\mathbf{Q}$ & Holladay I & SRK/T \\
\hline Mean predictive error $(D) \pm$ STD & $0.74 \pm 1.37$ & $0.66 \pm 1.41$ & $0.47 \pm 1.49$ \\
\hline Mean absolute predictive error (D) \pm STD & $1.20 \pm 0.99$ & $1.17 \pm 1.02$ & $1.09 \pm 1.11$ \\
\hline Median predictive error (D) & 0.718 & 0.719 & 0.449 \\
\hline Median absolute predictive error (D) & 0.919 & 0.881 & 0.775 \\
\hline Correct within $\pm 0.50 \mathrm{D}(\%)$ & 23.85 & 22.94 & 33.03 \\
\hline Correct within $\pm 1.00 \mathrm{D}(\%)$ & 53.21 & 55.05 & 60.55 \\
\hline Correct within $\pm 2.00 \mathrm{D}(\%)$ & 83.45 & 87.16 & 88.99 \\
\hline Results with Mean Optimized Constants & Hoffer $\mathbf{Q}$ & Holladay I & SRK/T \\
\hline Mean predictive error $(D) \pm$ STD & $-0.35 \pm 1.39^{\ddagger}$ & $-0.29 \pm 1.40^{\ddagger}$ & $-0.11 \pm 1.46^{\ddagger}$ \\
\hline Mean absolute predictive error $(D) \pm$ STD & $1.05 \pm 0.98$ & $1.03 \pm 1.00$ & $0.99 \pm 1.08$ \\
\hline Median predictive error (D) & -0.280 & -0.262 & -0.105 \\
\hline Median absolute predictive error (D) & 0.800 & 0.711 & 0.638 \\
\hline Correct within $\pm 0.50 \mathrm{D}(\%)$ & $35.77 \%$ & $38.53 \%$ & $42.20 \%$ \\
\hline Correct within $\pm 1.00 \mathrm{D}(\%)$ & $63.30 \%$ & $64.22 \%$ & $63.30 \%$ \\
\hline Correct within $\pm 2.00 \mathrm{D}(\%)$ & $88.07 \%$ & $85.32 \%$ & $86.24 \%$ \\
\hline Results with Median Optimized Constants & Hoffer Q & Holladay I & SRK/T \\
\hline Mean predictive error $(D) \pm$ STD & $-0.19 \pm 1.38^{\ddagger}$ & $-0.03 \pm 1.40^{\ddagger}$ & $-0.03 \pm 1.47^{\ddagger}$ \\
\hline Mean absolute predictive error $(D) \pm$ STD & $1.01 \pm 0.96 *$ & $0.98 \pm 0.99^{\dagger}$ & $0.99 \pm 1.08^{*}$ \\
\hline Median predictive error (D) & -0.121 & -0.003 & -0.004 \\
\hline Median absolute predictive error (D) & 0.718 & 0.640 & 0.609 \\
\hline Correct within $\pm 0.50 \mathrm{D}(\%)$ & $37.61 \%$ & $43.12 \%$ & $41.28 \%$ \\
\hline Correct within $\pm 1.00 \mathrm{D}(\%)$ & $59.63 \%$ & $62.39 \%$ & $63.30 \%$ \\
\hline Correct within $\pm 2.00 \mathrm{D}(\%)$ & $87.16 \%$ & $85.32 \%$ & $87.16 \%$ \\
\hline
\end{tabular}

Notes: $\mathrm{N}=109$ for this table. ${ }^{*} \mathrm{p}<0.05$ for comparison between standard constant and optimized constant (paired $t$-test). ${ }^{\dagger} \mathrm{p}<0.0 \mathrm{I}$ for comparison between standard constant and optimized constant (paired $t$-test). ${ }^{\ddagger} \mathrm{p}<0.00 \mathrm{I}$ for comparison between standard constant and optimized constant (paired $t$-test).

Table 5 Standard and Optimized Constants

\begin{tabular}{|l|l|l|l|}
\hline Constant & Hoffer Q pACD & Holladay I SF & SRK/T A Constant \\
\hline Manufacturer constant for endocapsular placement & 4.69 & 0.91 & 117.7 \\
Mean optimized constant for surgeon and scleral fixation & 5.69 & 1.79 & 118.56 \\
Median optimized constant for surgeon and scleral fixation & 5.54 & 1.55 & 118.44 \\
\hline
\end{tabular}

fixation precludes the possibility of lens dislocation into the vitreous cavity and allows the procedure to be performed by a single surgeon without uncontrolled needles in the eye.

Our series includes 121 consecutive eyes that underwent placement of a scleral fixated CT Lucia 602 lens using the modified Yamane technique described. This is a 3-piece monofocal aspheric lens composed of a silicone acrylate optic and polyvinylidene $\mathrm{C}$ loop haptics designed for endocapsular fixation. It has become a popular SFIOL due to the strength of the haptics and their resistance to breakage when flexed. The manufacturer's recommended
A constant is 117.7 (a converted pACD of 4.69, SF of 0.91 ), which was used for all pre-operative IOL calculations. This lens has an optimized A constant for Hill RBF of 118.40, quite close to our mean optimized A constant of 118.56 and our median A constant of 118.44. The difference in our optimized constants from the manufacturer's A constant, the Hill RBF optimized A constant, as well as the variability of constants between eyes, is likely attributable to surgical factors: such as the anterior-posterior placement of the sclerotomy sites (and therefore the lens optic), increased tension on the angulated haptics (that could cause anterior displacement of the lens optic and 


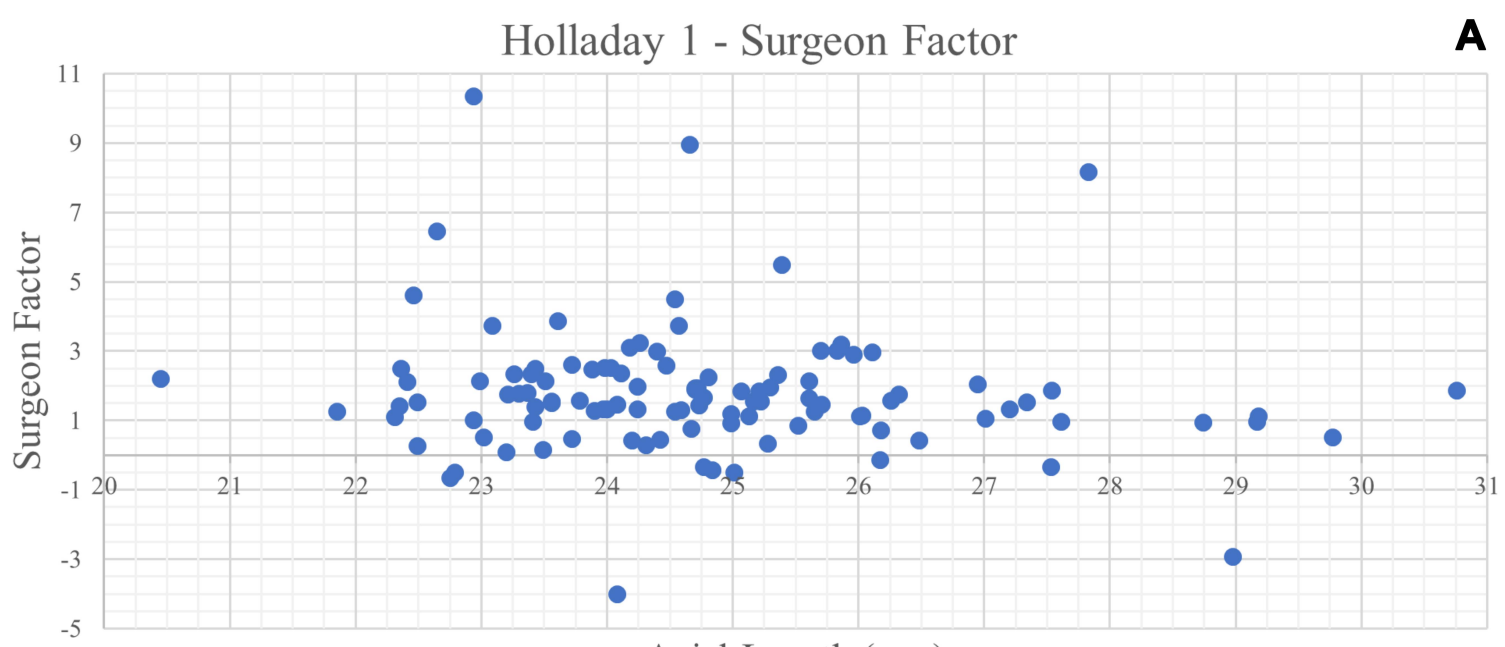

Axial Length (mm)

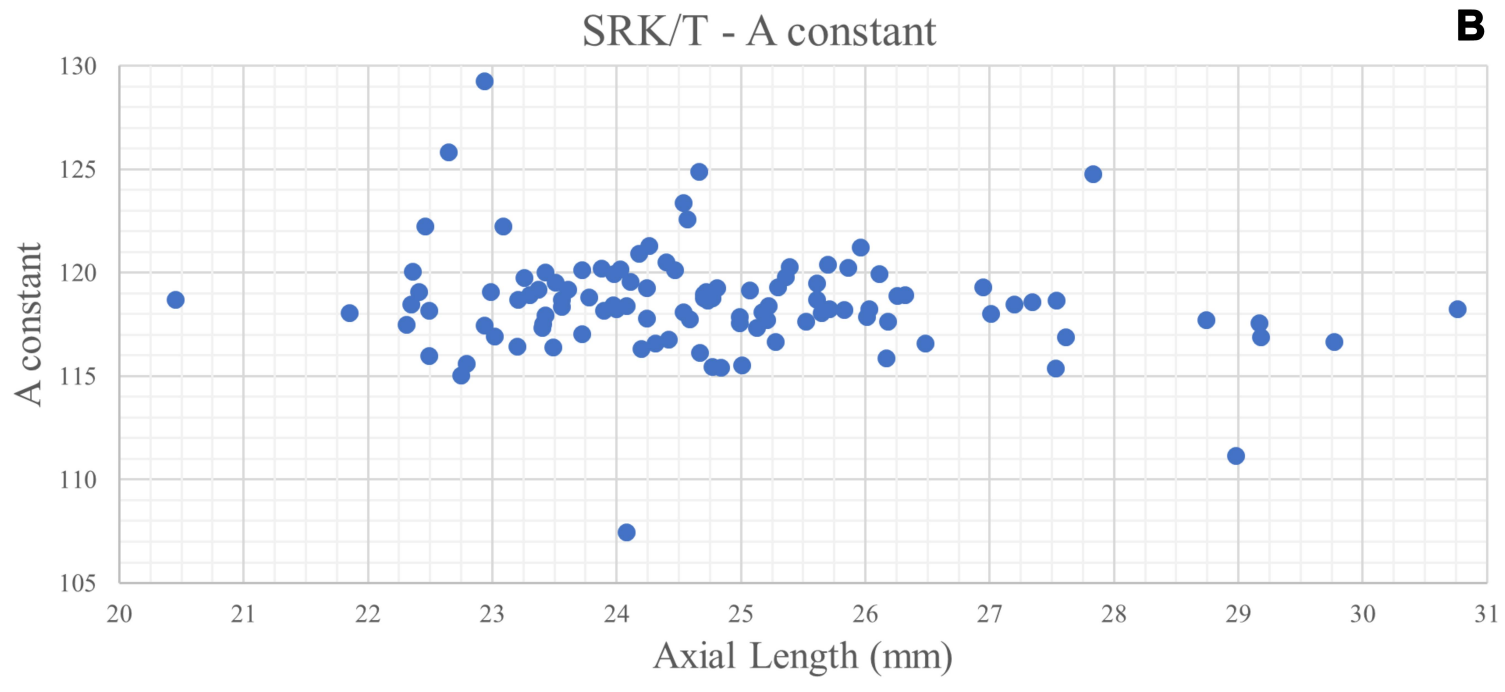

Hoffer Q - pACD

C

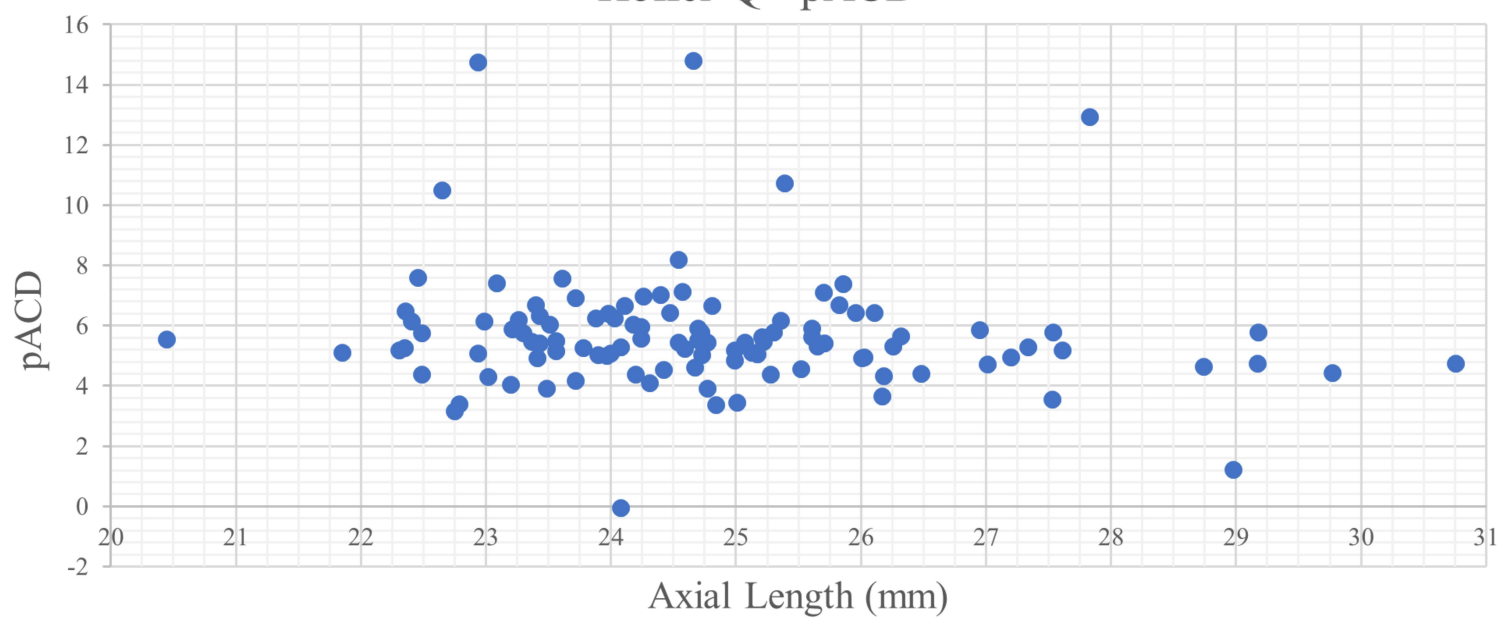

Figure I Scatter plots of axial length (in mm) versus (A) Surgeon Factor; (B) A- constant; (C) pACD with outliers excluded. 
a myopic shift), or a greater angle of approach of the sclerotomy needles (that could cause a posterior positioning of the lens or lens tilt resulting in a hyperopic shift or induced astigmatism, respectively). Using our mean optimized A constant should serve as a starting point and may reduce postoperative hyperopic refractive surprises. However, each surgeon should review his/her own results in order to further refine the lens constant. It is unlikely that our single needle modification would alter the refractive results. As demonstrated by Figure 1, the effective lens position for this technique is difficult to predict.

Our study included a heterogeneous group of patients with complicated past ocular histories and co-morbidities including trauma, ruptured globe, intraocular foreign body, retinal detachment, and corneal disease. In contrast to the Yamane et al series that did not report any vision-limiting outcomes, we report several serious postoperative complications including choroidal hemorrhage, corneal failure, atrophia bulbi, and endophthalmitis. Based on our experience with this technique, we do not believe these serious complications are due to our single-needle modification, but instead are a complication of SFIOLs in a more complex population.

Mastery of this technique is challenging, and excess haptic bend and stress may lead to suboptimal outcomes with varying degrees of lens decentration and tilt. Due to the sequential externalization of the haptics, it is possible to stretch and deform the leading haptic while withdrawing the trailing haptic, particularly if the latter is inserted too far into the lumen of the needle. This will lead to lens optic decentration and may be avoided by docking only the tip of the trailing haptic. Similarly, although the polyvinylidene haptics of the CT Lucia 602 lens are stronger and more flexible than ones made of polymethylmethacrylate (PMMA), the trailing haptic can still be deformed during insertion and docking leading to lens tilt and/or decentration. Lens tilt, which averaged 3.4 degrees in Yamane's series, may also occur as a result of an incorrect angle of approach or location while performing the $a b$ externo sclerotomies but should not be affected by using a single needle approach. This is, however, more likely to occur in a hypotonous globe, which can be deformed by external pressure from the needle pass. When performed together with a pars plana vitrectomy, the infusion cannula may be used to create a normotensive eye during this stage of the procedure. Elevating the intraocular pressure may also be used to tamponade a suprachoroidal or pars plicata hemorrhage if it were to occur intraoperatively.
Distal haptic protrusion may also occur despite efforts to bury the haptics for intrascleral fixation during surgery. Since the needle pass is transconjunctival, it is possible that a small amount of Tenon's capsule may occlude the ostium of the sclerotomy. In addition, haptic flanges have been shown to have significant variability in size and shape even though made of similar material. ${ }^{24}$ The polyvinylidene haptic of the CT Lucia 602 lens typically forms a mushroom-shaped dome when heated that may be larger than the more commonly used PMMA haptic or other similar lenses preventing consistent placement within a 30 -gauge sclerotomy tract. In many cases, the result is a trans-scleral fixated IOL rather than an intrascleral fixated IOL. We suspect this limitation predisposed our cases to more frequent haptic protrusion. Haptic tips that are clearly displaced and protruding beneath the conjunctiva can be successfully repositioned at the slit lamp under topical anesthesia and in sterile conditions after application of betadine using a blunt-tipped forceps or sterile cotton swab to avoid tearing the overlying conjunctiva. We found this approach prevented further protrusion and haptic exposure in 9 of our 11 eyes with this complication; the other 2 eyes required re-operation for surgical repositioning.

Reverse pupillary block occurred in 4 eyes in our series with one eye experiencing iris capture of the lens optic. Iris capture was noted in $8 \%$ of eyes by Yamane despite a prophylactic iridotomy in all cases. ${ }^{15}$ This finding, together with pigment dispersion, has also been reported previously in eyes undergoing trans-scleral sutured PCIOLs. ${ }^{25}$ The risk factors include an anteriorly displaced effective lens position, IOL decentration or tilt, iridodonesis, and young age. ${ }^{25,26}$ This complication might be avoided by surgical iridectomy or laser iridotomy, and leaving capsular remnants whenever possible during vitrectomy to support the iris-capsular diaphragm complex. In our cases, acute episodes were managed in-office with laser peripheral iridotomy ( 3 eyes) and/or sterile lens repositioning at the slit lamp with a 30 -gauge needle on a tuberculin syringe to release optic capture. Due to its infrequent occurrence, we do not think incisional iridectomy or laser iridotomy need to be performed for all cases.

This study represents a large case series of a single lens fixated to the sclera using a modified single-needle Yamane technique. This change precludes the need for a skilled surgical assistant. Similar to Yamane et al, we found significant improvement in postoperative visual acuity. However, in this heterogenous group of patients, the average visual acuity did not return to normal due, in large part, to pre-existing corneal and vitreoretinal disease. Eyes requiring scleral fixation of 
a posterior chamber lens have altered anterior segment anatomy possibly due to trauma, zonulopathy, scarring, or prior surgery. As noted in our series, these eyes may also have vision limiting comorbidities and should not be considered simply aphakic or needing only pseudophakic correction in order to obtain best corrected visual acuity. Although the majority of the post-operative complications reported here are transient and can be managed in clinic with readily available topical medications, we do report a few severe complications not previously noted, including corneal edema, choroidal hemorrhage, endophthalmitis, retinal detachment, and atrophia bulbi. Our complication rate may reflect the complex preoperative pathology in this series of eyes.

The strength of this study is three-fold: (1) it is a large series of consecutive procedures, (2) it was performed by a single surgeon, and (3) all cases used the same lens. This study is limited by its retrospective nature and inclusion of eyes with complicated past ocular histories, which adds additional variability to the visual acuity, but not the refractive outcomes. Furthermore, some eyes have a relatively short follow-up period, and there were no objective measurements for intraocular lens tilt.

\section{Conclusion}

We feel our modified Yamane technique represents a simplification of the previous method given its ability to be performed safely without a surgical assistant. It provides an excellent method for posterior chamber lens implantation in patients without capsular support. Additionally, we report a mean optimized pACD of 5.69, Surgeon Factor of 1.79, and A constant of 118.56 for use with scleral fixation of the CT Lucia 602 lens using our method. This technique provides very good visual acuity in a heterogenous population of eyes, but may be associated with vision-threatening complications in some cases.

\section{Acknowledgments}

The authors would like to thank our vitreoretinal surgeons, Drs. Baseer U. Ahmad, Thomas B. Connor, Jonathan P. Greenberg, Dennis P. Han, Judy E. Kim, Raafay Sophie, David V. Weinberg, and William J. Wirostko. A special thank you to Dr. Warren Hill for his assistance with lens constant optimization. This work was supported by a gift from Dr. Myrna Larson and Dr. Nelson A. Moffat. Additionally, this work was supported by National Institutes of Health (EY014800), and an Unrestricted Grant from Research to Prevent Blindness, New York, NY, to the Department of Ophthalmology \&
Visual Sciences, University of Utah. The sponsor or funding organization had no role in the design or conduct of this research.

\section{Disclosure}

Dr. Eileen S. Hwang reports grants from Research to Prevent Blindness, National Institutes of Health, during the conduct of the study; non-financial support from Alcon, Bausch \& Lomb, Beaver Vistec, Katalys, Regeneron, and Spark Therapeutics, outside the submitted work. The authors report no other conflicts of interest in this work.

\section{References}

1. Por YM, Lavin MJ. Techniques of intraocular lens suspension in the absence of capsular/zonular support. Surv Ophthalmol. 2005;50 (5):429-462. doi:10.1016/j.survophthal.2005.06.010

2. Moschos MM, Nitoda E. The correction of aphakia using anterior chamber intraocular lens. In Vivo. 2016;30(6):733-738. doi:10.21873/invivo.10988

3. Ellingson FT. The uveitis-glaucoma-hyphema syndrome associated with the mark viii anterior chamber lens implant. $J$ Am Intraocul Implant Soc. 1978;4(2):50-53. doi:10.1016/S0146-2776(78)80054-8

4. Apple DJ, Mamalis N, Loftfield K, et al. Complications of intraocular lenses. A historical and histopathological review. Surv Ophthalmol. 1984;29(1):1-54. doi:10.1016/0039-6257(84)90113-9

5. Hannush SB. Sutured posterior chamber intraocular lenses: indications and procedure. Curr Opin Opthalmol. 2000;11(4):233-240. doi:10.1097/00055735-200008000-00004

6. Brunin G, Sajjad A, Kim EJ, et al. Secondary intraocular lens implantation: complication rates, visual acuity, and refractive outcomes. J Cataract Refract Surg. 2017;43(3):369-376. doi:10.1016/j.jcrs.2016.12.024

7. Buckley EG. Hanging by a thread: the long-term efficacy and safety of transscleral sutured intraocular lenses in children (an American Ophthalmological Society thesis). Trans Am Ophthalmol Soc. 2007;105:294-311.

8. Vote BJ, Tranos P, Bunce C, Charteris DG, Da Cruz L. Long-term outcome of combined pars plana vitrectomy and scleral fixated sutured posterior chamber intraocular lens implantation. Am J Ophthalmol. 2006;141(2):308-312. doi:10.1016/j.ajo.2005.09.012

9. Werner L, Wilbanks G, Nieuwendaal CP, et al. Localized opacification of hydrophilic acrylic intraocular lenses after procedures using intracameral injection of air or gas. J Cataract Refract Surg. 2015;41 (1):199-207. doi:10.1016/j.jcrs.2014.10.025

10. Balendiran V, MacLean K, Mamalis N, Tetz M, Werner L. Localized calcification of hydrophilic acrylic intraocular lenses after posterior segment procedures. J Cataract Refract Surg. 2019;45 (12):1801-1807. doi:10.1016/j.jcrs.2019.07.015

11. Gabor SGB, Pavlidis MM. Sutureless intrascleral posterior chamber intraocular lens fixation. J Cataract Refract Surg. 2007;33 (11):1851-1854. doi:10.1016/j.jcrs.2007.07.013

12. Matsui Y, Matsubara H, Hanemoto T, Kondo M. Exposure of haptic of posterior chamber intraocular lens after sutureless intrascleral fixation. BMC Ophthalmol. 2015;15(1):102-104. doi:10.1186/ s12886-015-0102-3

13. Kumar DA, Agarwal A, Packiyalakshmi S, Jacob S, Agarwal A. Complications and visual outcomes after glued foldable intraocular lens implantation in eyes with inadequate capsules. J Cataract Refract Surg. 2013;39(8):1211-1218. 
14. Scharioth GB, Prasad S, Georgalas I, Tataru C, Pavlidis M. Intermediate results of sutureless intrascleral posterior chamber intraocular lens fixation. $J$ Cataract Refract Surg. 2010;36 (2):254-259. doi:10.1016/j.jcrs.2009.09.024

15. Yamane S, Sato S, Maruyama-Inoue M, Kadonosono K. Flanged intrascleral intraocular lens fixation with double-needle technique. Ophthalmology. 2017;124(8):1136-1142. doi:10.1016/j.ophtha.2017 .03 .036

16. Jacob S, Kumar DA, Rao NK. Scleral fixation of intraocular lenses. Curr Opin Ophthalmol. 2020;31(1):50-60. doi:10.1097/ICU.0000000 000000632

17. Hwang ES, Warren CC, Koenig SB. Flanged intrascleral intraocular lens fixation with a single needle. J Cataract Refract Surg. 2018;44 (12):1526-1527. doi:10.1016/j.jcrs.2018.08.020

18. Yamane S, Maruyama-Inoue M, Kadonosono K. Needle stabilizer for flanged intraocular lens fixation. Retina. 2019;39(4):801.

19. Abbey AM, Hussain RM, Shah AR, Faia LJ, Wolfe JD, Williams GA. Sutureless scleral fixation of intraocular lenses: outcomes of two approaches. The 2014 yasuo tano memorial lecture. Graefe's Arch Clin Exp Ophthalmol. 2015;253(1):1-5. doi:10.1007/ s00417-014-2834-9

20. Rocke JR, McGuinness MB, Atkins WK, et al. Refractive outcomes of the Yamane flanged intrascleral haptic fixation technique. Ophthalmology. 2020;127(10):1429-1431. doi:10.1016/j.ophtha.2020 .03 .032
21. Hoffer KJ. The Hoffer Q formula: a comparison of theoretic and regression formulas. J Cataract Refract Surg. 1993;19(6):700-712. doi:10.1016/S0886-3350(13)80338-0

22. Retzlaff JA, Sanders DR, Kraff MC. Development of the SRK/T intraocular lens implant power calculation formula. J Cataract Refract Surg. 1990;16(3):333-340. doi:10.1016/S0886-3350(13) 80705-5

23. Holladay JT, Musgrove KH, Prager TC, Lewis JW, Chandler TY, Ruiz RS. A three-part system for refining intraocular lens power calculations. J Cataract Refract Surg. 1988;14(1):17-24. doi:10.1016/S0886-3350(88)80059-2

24. Kronschläger M, Blouin S, Roschger P, Varsits R, Findl O. Attaining the optimal flange for intrascleral intraocular lens fixation. J Cataract Refract Surg. 2018;44(11):1303-1305.

25. Bang SP, Joo CK, Jun JH. Reverse pupillary block after implantation of a scleral-sutured posterior chamber intraocular lens: a retrospective, open study. BMC Ophthalmol. 2017;17(1):1-8. doi:10.1186/s12886-017-0427-1

26. Choi SR, Jeon JH, Kang JW, Heo JW. Risk factors for and management of pupillary intraocular lens capture after intraocular lens transscleral fixation. J Cataract Refract Surg. 2017;43(12):1557-1562. doi:10.1016/j.jcrs.2017.08.021
Clinical Ophthalmology

\section{Publish your work in this journal}

Clinical Ophthalmology is an international, peer-reviewed journal covering all subspecialties within ophthalmology. Key topics include: Optometry; Visual science; Pharmacology and drug therapy in eye diseases; Basic Sciences; Primary and Secondary eye care; Patient Safety and Quality of Care Improvements. This journal is indexed on PubMed

\section{Dovepress}

Central and CAS, and is the official journal of The Society of Clinical Ophthalmology (SCO). The manuscript management system is completely online and includes a very quick and fair peer-review system, which is all easy to use. Visit http://www.dovepress.com/ testimonials.php to read real quotes from published authors. 